ORIGINAL RESEARCH

\author{
G.M. Karwacki \\ J.F. Schneider
}

\section{Normal Ossification Patterns of Atlas and Axis: A CT Study}

BACKGROUND AND PURPOSE: Development of the CVJ is a complex process rarely analyzed by CT. Cartilaginous remnants within the atlas and axis have been shown to variably persist throughout childhood and may be mistaken for fractures. The purpose of this study was to better estimate the fusion timeline of the synchondroses at the CVJ.

MATERIALS AND METHODS: We retrospectively reviewed singular CT scans in 550 children without known skeletal dysplasia or maturation delay (from neonate to 17 years) and analyzed the ossification of atlas and axis. Normal closing age for synchondroses was defined as soon as a complete ossification rate of $80 \%$ or more per age category was achieved.

RESULTS: No separate OC was observed in 38 of 230 incompletely ossified AAAs, whereas single, bipartite, or multiple OCs were observed in, respectively, 196, 86, and 16 of 298 AAAs in which OCs could be identified. Synchondroses at the AAA closed after 12 years (range 4.5-17 years). Posterior midline synchondrosis of the atlas closed after 4 years (range 2-13 years). The axis ossified from 6 OCs and 4 synchondroses. Subdental and neurocentral synchondroses closed simultaneously after 9 years (range 7-9.5 years). Apicodental synchondrosis and chondrum terminale were completely ossified after 10.5 years (range $5.5-13.5$ years).

CoNCLUSIONS: Analysis of the CVJ using triplanar CT reconstructions allows secure identification of characteristic developmental features of the atlas and axis, and helps to separate normal variants from true osseous lesions.

ABBREVIATIONS: $\mathrm{AAA}=$ anterior atlas arch; $\mathrm{ADS}=$ apicodental synchondrosis; $\mathrm{CHT}=$ chondrum terminale; $\mathrm{CVJ}=$ craniocervical junction; $\mathrm{OC}=$ ossification center; $\mathrm{PAA}=$ posterior atlas arch; SDS $=$ subdental synchondrosis
$\mathbf{T}$ he CVJ is the most common site of osseous and ligamentous injuries in pediatric cervical spine trauma. ${ }^{1}$ Because of the complex cervical vertebral embryology, some normal variations, such as supplementary open synchondroses or atypical form, location, or number of OCs, may be misread as fractures.

Knowledge of normal pre- and postnatal cervical spine maturation has been derived mainly from autopsy specimens and plain film imaging ${ }^{2-6}$ and only rarely by CT. ${ }^{7,8}$ The limited ability of children to cooperate in obtaining satisfactory roentgenograms has established CT as the technique of choice to study acute CVJ injuries. ${ }^{9}$ The presence of large unossified cartilaginous parts of the atlas and axis, numerous and sometimes unexpected synchondroses, and osseous gaps at a laterthan-expected age, are potential pitfalls. Recent work has attempted to estimate the ossification rate of normal synchondroses at the CVJ. ${ }^{8}$ Nevertheless, variations within the anterior part of the atlas and at the apical portion of the dens need further analysis. Therefore, the aim of our study was to more extensively determine the normal milestones in the osseous development of CVJ in CT images of children and adolescents, paying special attention to time-related closure of synchon-

Received January 9, 2012; accepted after revision February 13.

From the Departments of Radiology (G.M.K.) and Pediatric Radiology (G.M.K., J.F.S.), University Hospital Basel, Basel, Switzerland.

Please address correspondence to J.F. Schneider, MD, Head Pediatric Radiology, University Children's Hospital UKBB, CH-4005 Basel, Switzerland; e-mail: jacques.schneider@ukbb.ch

Indicates open access to non-subscribers at www.ajnr.org

http://dx.doi.org/10.3174/ajnr.A3105 droses and osseous bridges, and to identify normal variants, based on a large population.

\section{Materials and Methods}

This retrospective study included all 933 consecutive studies of children who underwent a CT examination of the head and neck at our institution between July 2003 and October 2007. The study was approved by the local ethics committee. Exclusion criteria were incomplete visualization of the CVJ, radiologic evidence of traumatic lesion, known skeletal dysplasia, and maturation delay. We reviewed a total of 550 patients, and their scans were retrieved through our PACS.

\begin{tabular}{lc}
\hline Number of CT examinations per age category & \\
\hline Age (yrs) & Patients \\
\hline 0 & 46 \\
1 & 38 \\
2 & 35 \\
3 & 36 \\
4 & 31 \\
5 & 31 \\
6 & 25 \\
7 & 25 \\
8 & 33 \\
9 & 28 \\
10 & 30 \\
11 & 26 \\
12 & 39 \\
13 & 41 \\
14 & 41 \\
15 & 35 \\
16 & 6 \\
17 & 4 \\
Total & 550 \\
\hline
\end{tabular}



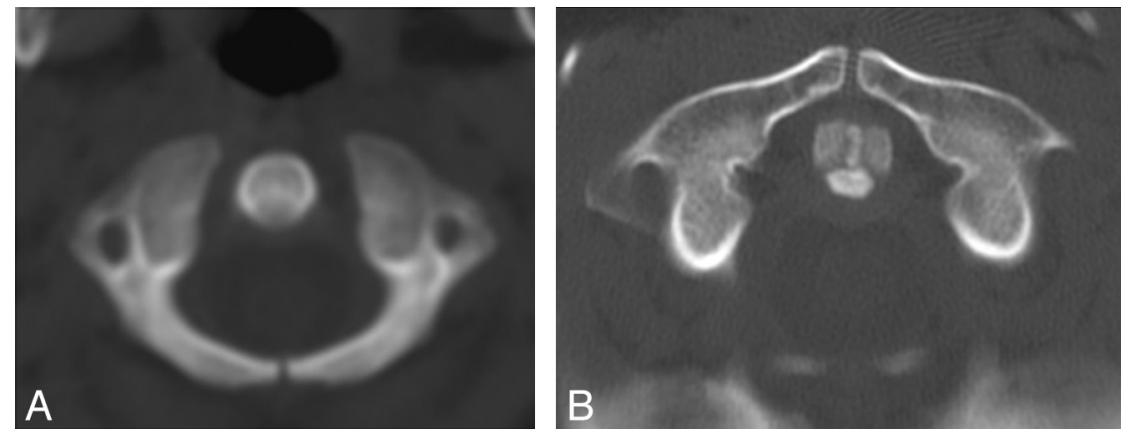

Fig 1. Two examples of absence of ventral ossification center of the AAA. $A$, Large nonossified AAA in a 2-year-old child. $B$, Midline cartilaginous gap at the AAA in a 12-year-old child.
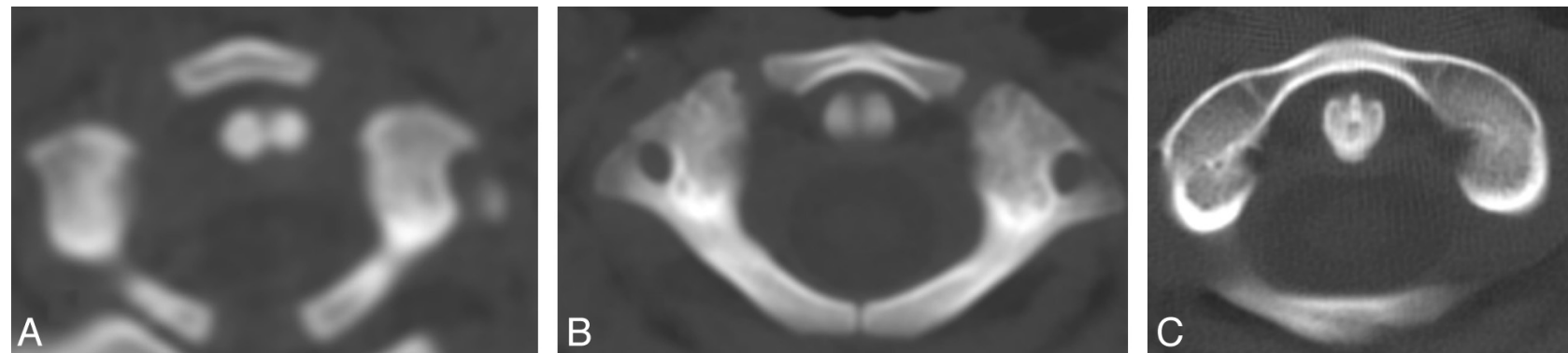

Fig 2. Singular midline $\mathrm{OC}$ pattern at the AAA. A, 5-month-old child with already large midline $\mathrm{OC}$ and large ventrolateral synchondroses. $B$, A 2.5-year-old child with progressive ossification of the chondral AAA anlage from both the central $\mathrm{OC}$ and the lateral masses of the atlas. $C, 7$-year-old child with complete ossification of the AAA but still-visible ventrolateral synchondroses.
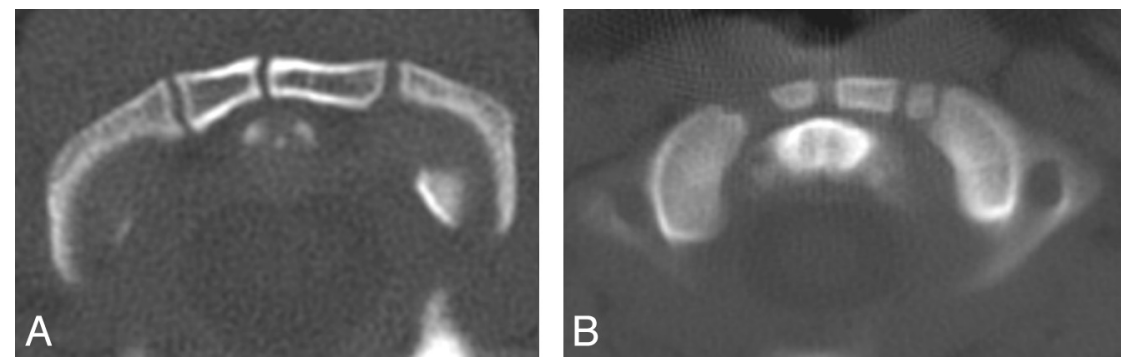

Fig 3. Multiple OC patterns at the AAA. A, 3-year-old child with 2 OCs paramedially located. B, A 2.5-year-old child with 3 irregular OCs within the AAA.

\section{Ossification of ventral atlas arch}

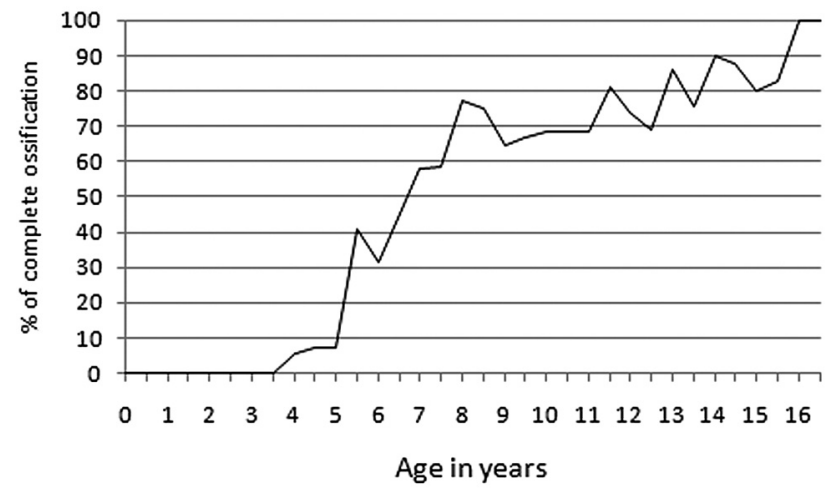

Fig 4. Ossification timetable of the anterior atlas arch.

There were 230 girls ( $42 \%$ ) and 320 boys (58\%), age range 2 days to 17 years, with a median age of 8 years (Table 1). In the case of multiple examinations per patient, only the first scan was considered. The examinations were performed on a 16-section scanner (Brilliance; Philips, Best, the Netherlands). Primary acquisition in the axial plane was completed in soft algorithm, with a maximal section thickness of $3 \mathrm{~mm}$. All studies were reconstructed with a bone algorithm in the axial plane. CVJ was assessed using these axial bone images, as well as their reformations in other planes, when available. In 274 patients, reformations in all 3 planes were performed (50\%). In 65 patients, there were only axial and coronal reformation planes (12\%), whereas axial and sagittal reformation planes only were available in 30 patients (5\%). In 181 children, CVJ was estimated based on axial scans only (33\%). After the ossification status of the OCs and the synchondroses was analyzed, patients were grouped into 6-month-interval age categories. The rate of complete ossification was assessed as a percentage of the total observations per each age category.

\section{Results}

\section{Ossification of the Atlas}

AAA. Ossification of the AAA was assessed in 546 patients. It proceeded, on one hand, from the lateral masses, and, on the other hand, from OCs appearing within the ventral cartilaginous band.

In 230 of 546 patients ( $42 \%$ ), the AAA was partially ossified 

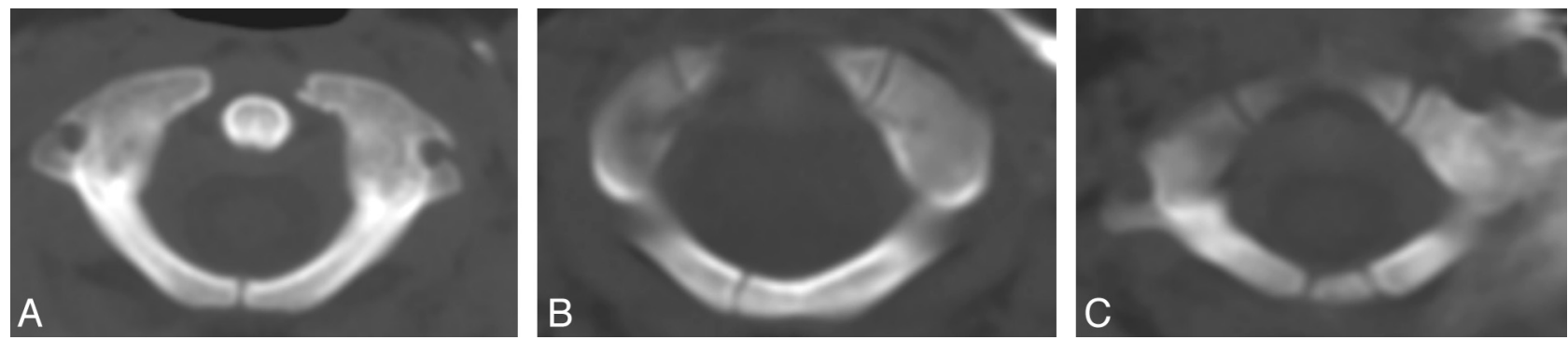

Fig 5. Different aspects of the PAA. $A$, Normal midline synchondrosis. $B$, Rare paramedial posterior synchondrosis. $C$, Rare midline $0 C$ within the PAA.

Ossification of posterior atlas arch

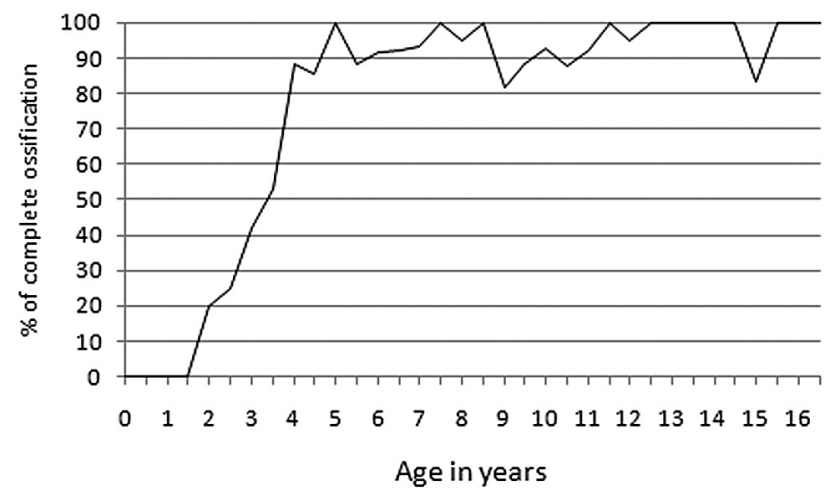

Fig 6. Ossification timetable of the posterior atlas arch.

(age range 2 days to 9 years) and ossification followed the shape of the AAA anlage to stop at the ventrolateral synchondrosis. In 38 of 230 patients (16.5\%), a large unossified midline gap and no ventral OC were observed (age range $0-12$ years; Fig 1).

In 316 of 546 patients (58\%), the AAA was already completely ossified (age range 4.5-17 years), but in 106 of these 316 patients, fusion line remnants allowed adequate assessment of previous OCs. Hence, ossification centers could be assessed in 298 patients and we observed a singular OC in 196 patients (66\%; Fig 2), bipartite OCs in 86 patients (29\%; Fig $3 A$ ), and multiple OCs in 16 patients (5\%; Fig $3 B$ ). Complete ossification of the AAA was observed in over $80 \%$ of children at the approximate age of 12 years (age range 4.5-17 years; Fig 4).

PAA. Ossification of the PAA was assessed in 544 patients. It proceeded from the posterior parts of the osseous hemiarches along the cartilaginous band stretching between. A posterior gap was usually situated in the midline, but a minimal lateral shift was not uncommon, though clearly asymmetric closure was exceptional (Fig $5 A,-B)$. In 3 patients $(0.5 \%$ ), we observed an additional, centrally located OC within the PAA (Fig 5C). Complete ossification of the PAA was observed in over $80 \%$ of children at the approximate age of 5 years (age range 2-13 years; Fig 6). In children older than 5 years (372 patients), the PAA was fully ossified in 352 patients (95\%) and only sporadic minimal slits were observed.

\section{Ossification of the Axis}

Ossification of the axis was assessed in 546 patients. The axis consisted of 4 synchondroses and 6 ossification centers. Two vertically oriented neurocentral synchondroses separated the centrum from the lateral neural arches. Two horizontal synchondroses di-
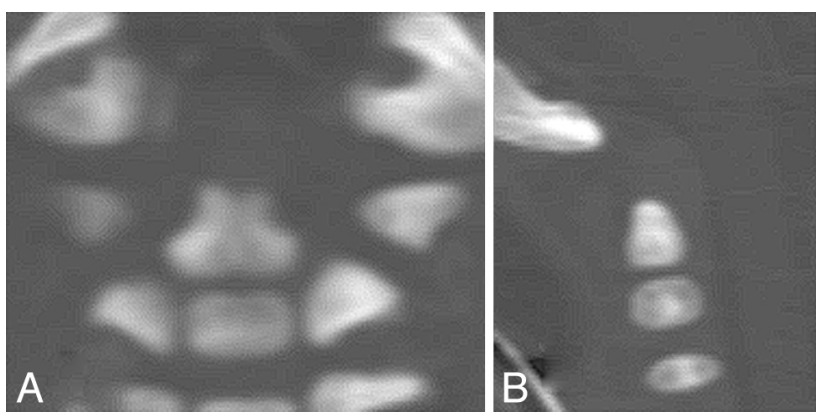

Fig 7. Coronal $(A)$ and sagittal $(B)$ view of the neonatal axis with $5 \mathrm{OCs}$. $A$, While both club-shaped centers of the dens are already fused at birth, a midline vertical fusion line can still be perceived. $B$, Smooth cartilaginous rounded $\mathrm{CHT}$ at the top of the dens is best appreciated on the sagittal view.

Ossification of subdental synchondrosis

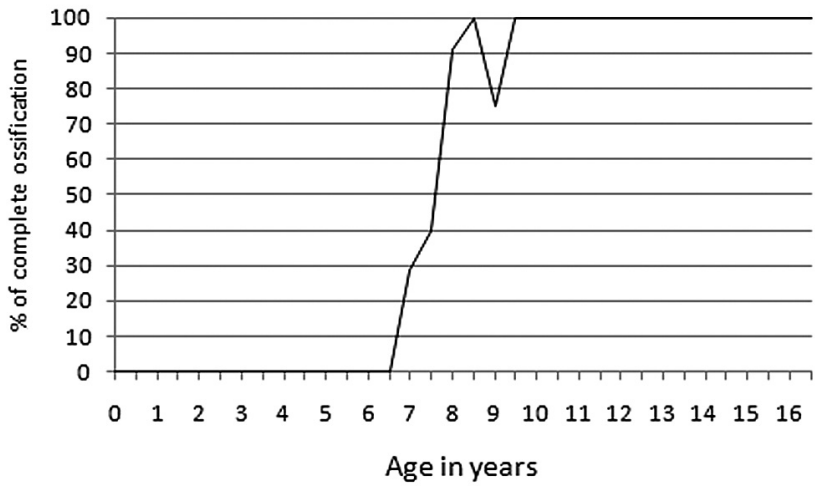

Fig 8. Ossification timetable of the subdental synchondrosis

vided the central column of the axis: 1) the SDS separated the centrum from the twin OCs of the dens above, and 2) the ADS had a V-shaped appearance on coronal view and separated the twin OCs of the dens from the CHT at the tip.

Five OCs were already partially ossified at birth: 1) $1 \mathrm{OC}$ for the centrum, 2) 2 OCs for the lateral neural arches, 3) 2 OCs for the dens, above the centrum, which were already fused in the midline, and 4) a singular OC for the CHT observed crowning the dens (Fig 7).

The SDS and neurocentral synchondrosis could be adequately assessed in 256 children, of which 112 were incompletely ossified (44\%). Both synchondroses demonstrated a similar and rapid ossification, which was completed in over $80 \%$ of children at the approximate age of 9 years (age range 7-9.5 years; Fig 8). A central hyperattenuated ossification spot developed in the middle of the SDS between 3 years and 7 years, and still remained clearly visible well after complete occlusion of the SDS (Fig 9). 

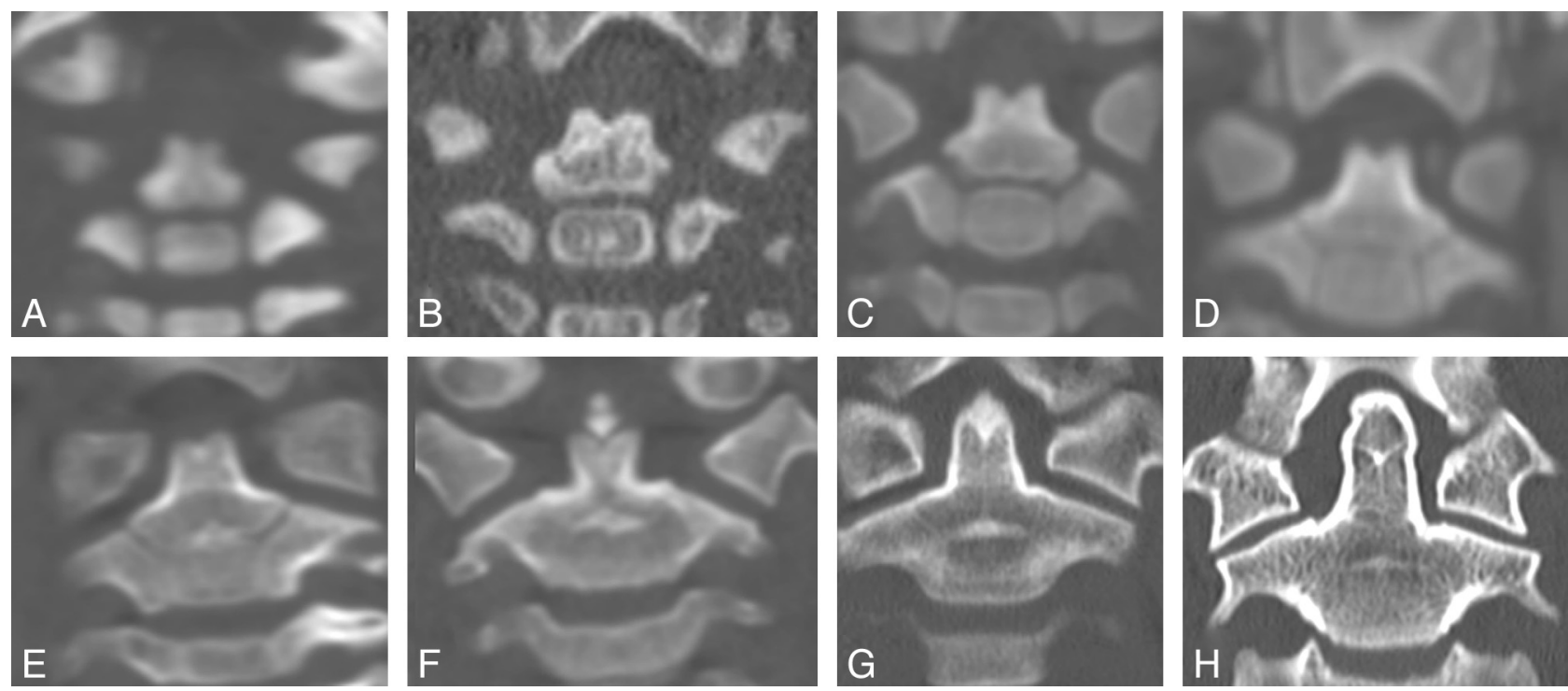

Fig 9. Coronal view of ossification development of the axis. Children are of the following ages $(A) 30$ days, $(B) 5$ months, $(C) 1.5$ years, $(D) 2$ years, $(E) 3$ years, $(F) 5$ years, $(G) 8.5$ years, and $(H) 14$ years.

Ossification of apicodental synchondrosis

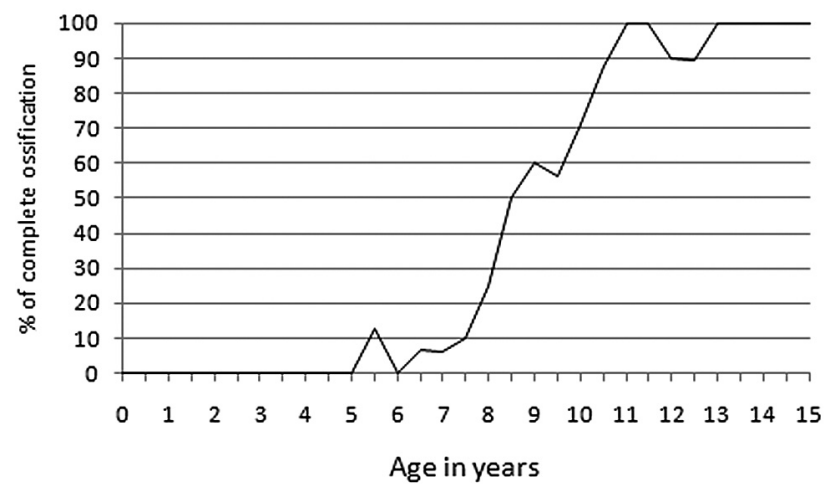

Fig 10. Ossification timetable of the apicodental synchondrosis.

The ADS and CHT could be adequately assessed in 546 patients. A central hyperattenuated ossification spot was initially observed within the ADS in 184 patients $(34 \%)$ and eventually became integrated in the dens (age range $1.5-13$ years). A separate OC in the CHT was observed in 49 patients (9\%) and arose in a centroposterior location in 37 patients $(75 \%)$, in a solitary anterior location in 3 patients (7\%), and was multifocal in 7 patients (16\%). Ossification of the dens apex left a stellate fusion line in axial and coronal planes (Fig $9 H$ ). ADS and CHT were completely ossified in over $80 \%$ of children at the approximate age of 10.5 years (age range 5.513.5 years; Fig 10$)$.

\section{Discussion}

Our study shows that there is a general caudal to cranial gradient in the growth of ossification centers and closure of synchondroses in the atlas and axis. Closure of the horizontally oriented medial synchondroses began at the base of the dens at the SDS and ended at the AAA. On the other hand, the vertically oriented lateral neurocentral synchondrosis fused at an age similar to the SDS, which is in line with postmortem radiologic evaluation, ${ }^{2,6}$ but slightly later compared with a pediatric population examined by MR imaging, in which these closed at approximately 7 years. ${ }^{10}$ This is possibly because MR imaging was used instead of CT, which delivers a lower anatomic resolution and has an inherent weakness in depicting bone structure.

Considering the AAA, we demonstrate a larger variability in ossification patterns compared with data in the literature. ${ }^{8,11-13}$ The prevalence of an anterior midline synchondrosis has been estimated to be as low as $20 \%,{ }^{8}$ but the presence of bipartite or multiple OCs and, hence, an anterior midline synchondrosis was identified in our study in 102 patients (34\%). It has been postulated that the $\mathrm{OC}$ in the anterior arch of atlas develop through intramembranous perichondral ossification, probably as a result of the forces applied by the anterior longitudinal ligament. ${ }^{14}$ Because ossification centers require a blood supply, the number of developing OCs may be also determined by the vascularization pattern. On the other hand, the variable number of ossification centers seemed to affect neither the closure time of synchondroses nor the general shape of AAA. If no ossification centers develop within the AAA, osseous extensions spread ventrally directly from the lateral masses. This pattern was identified in 38 patients $(16.5 \%)$ in our series, much higher in comparison with the literature $(0.7 \%){ }^{8}$ Nevertheless, no persistent ventral cleft was found after the estimated closure age of 12 years, possibly because of the low incidence of these gaps, which was found to be as low as $0.1 \%$ in an autopsy study. ${ }^{15}$ Moreover, our study shows that $80 \%$ of the patients had a complete ossification pattern after the age of 12 years, which was higher than in the literature, in which a median age between 7 and 8.5 years has been described. ${ }^{7,8}$ Persistent gaps at the posterior arch were found in approximately $4 \%$ in the same autopsy study, ${ }^{15}$ while, in our study, only minimal slits could be found.

Last, congenital defects, though some may assume bizarre forms, are usually easy to differentiate from acute injury on the basis of their sclerotic margins. Furthermore, our study also confirms that, in all cases, these osseous gaps are actually unossified fibrous tissue bridges; therefore, stability is not an issue. $^{16}$ 

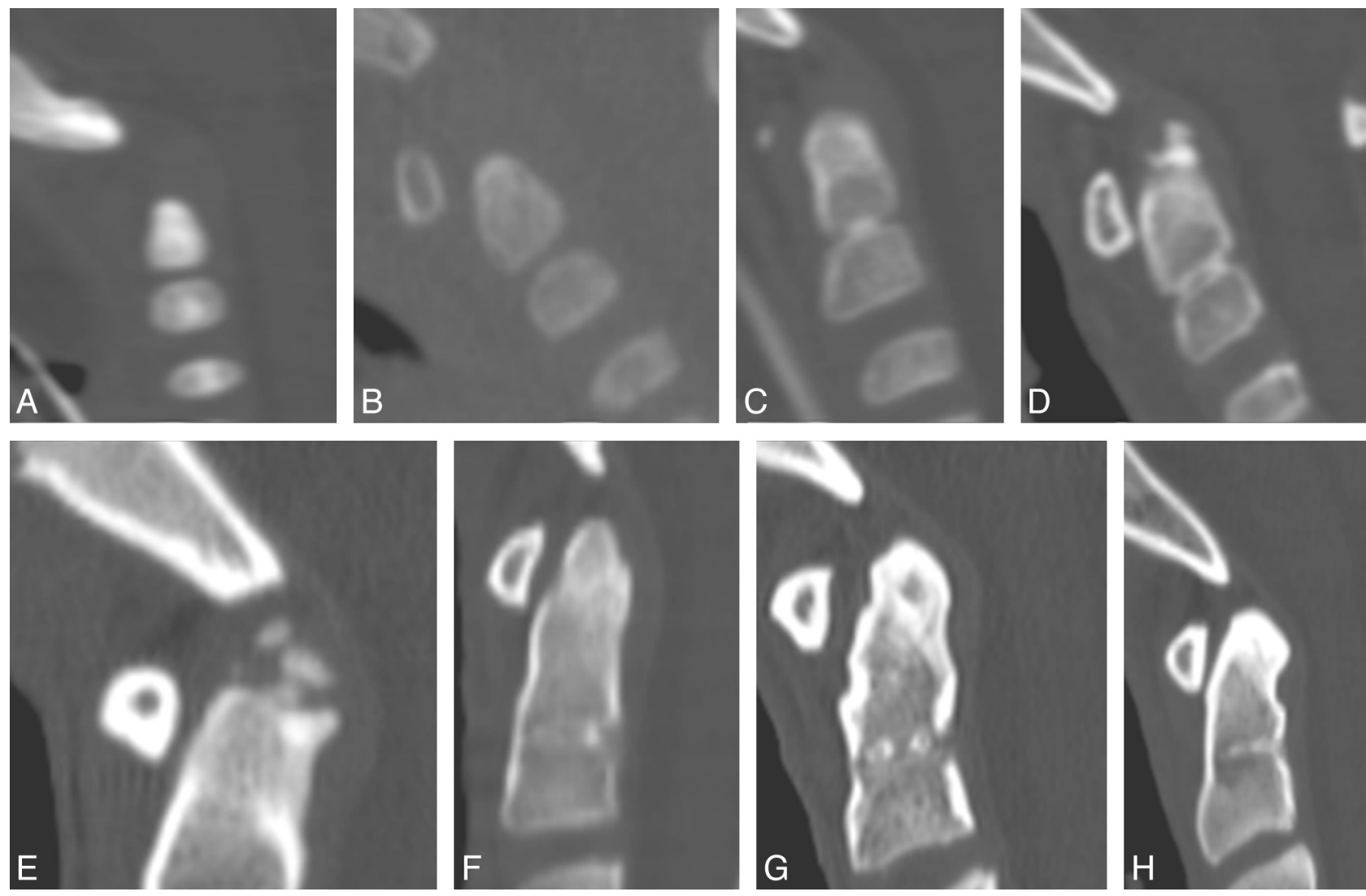

Fig 11. Sagittal view of ossification development of the axis. Children are of the following ages: $(A) 30$ days, $(B) 3$ months, $(C) 3$ years, $(D) 5$ years, $(E) 6.5$ years, $(F) 8$ years, $(G) 13$ years, and $(H) 16$ years.

Ossification of the axis has been described in numerous publications. $^{5,6,17}$ Unresolved and controversially discussed are the origin of the odontoid process and the etiology of an os odontoideum, both topics being beyond the scope of this article. ${ }^{18-20}$ Furthermore, ossification patterns of the ADS and the tip of the dens have not been analyzed in similar studies. ${ }^{8}$ Most relevant in clinical practice are the appearance and closure of synchondroses in the central axis column and the ossification evolution of the axial tip. The SDS, located below the superior articular facets of the axis, is the preferred plane of fracture in children. ${ }^{21,22}$ At a histologic level, a chondroid matrix can be identified, which represents the remnant of an intervertebral disk blastema. ${ }^{23}$ Consequently, healing within this physis and focal osseous bridging may provide stability at the expense of growth potential, though longitudinal growth of C2 occurs mainly at the ADS level and only marginally at the SDS level.

Both the SDS and ADS show a similar central ossification spot at the beginning of the ossification process on both coronal and sagittal views (Figs 9 and 11). Analogous to the SDS, one can postulate that the ADS also represents a small residual disk blastema, which will be eventually completely resorbed and assimilated into the CHT. One can also postulate that the ADS is a further line of weakness within the axis. Although dens fractures in children preferentially occur through the physis of the SDS, traumatic forces applied at the upper part of the dens could result in injuries of the ADS. Dislocations at that level in early childhood may explain why a large ossiculum terminale or even an os odontoideum may develop from the CHT, in which the arterial supply through the apical ligament is preserved. Furthermore, associated dens hypoplasia, in which arterial supply and, hence, consecutive growth are disturbed, may occur at the same time, as has been previously described in the literature. ${ }^{24,25}$

The last part of the axis to ossify is the CHT, and its ossification pattern was most variable. ADS and CHT were completely ossified in over $80 \%$ of children at the approximate age of 10.5 years, which was slightly higher than that reported in the literature, with a median age of 8.2 years. ${ }^{8}$ Multiple ossification centers within the CHT and an asymmetric appearance may sometimes mimic osseous posttraumatic fragments, but a distinction can be made when considering the smooth surrounding cartilaginous envelope covering the OC itself, the surface of which was in regular continuity with the dens below (Fig 12). A completely separate ossification can grow quite large and may not fuse with the ADS and OCs of the dens, leading to a persistent ossiculum terminale (Fig 13). This is generally of little clinical significance, as it lies above the transverse ligament and its position does not change during movements at the CVJ. ${ }^{14,19}$

The current study has several shortcomings. There was no analysis of variation by sex or race. As a retrospective study, technical parameters were not homogeneous in all examinations and, especially, triplanar reconstructions were available in only $50 \%$ of cases, while biplanar reconstructions were retrieved in $17 \%$. Assessment of synchondroses and OCs in the axial plane only (33\%) may have led to incorrect classification. From a study design point of view, a longitudinal approach 

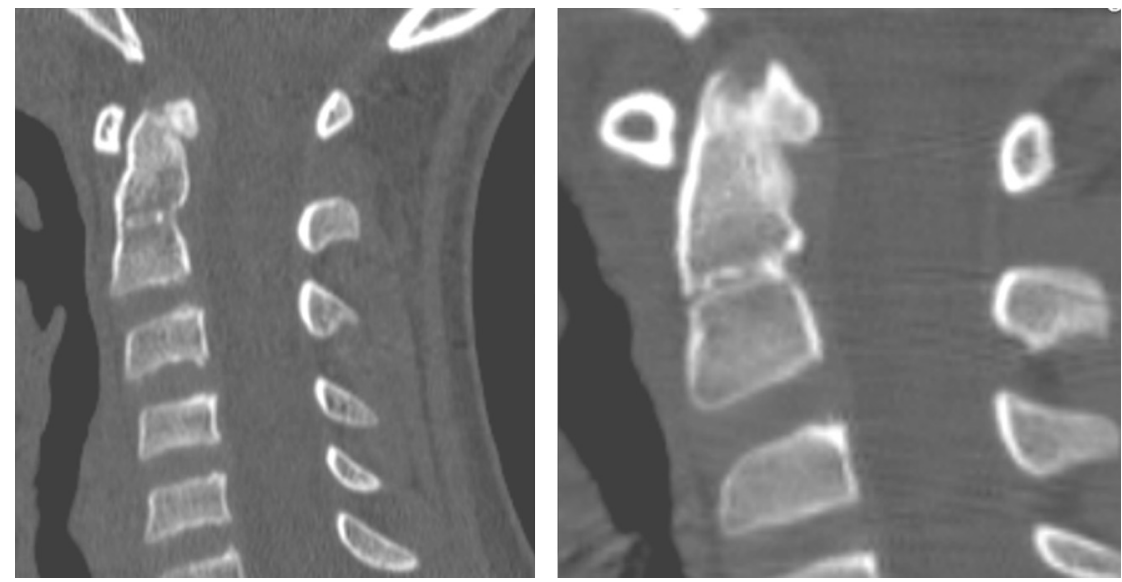

Fig 12. Rare posterior ossification of the $\mathrm{CHT}$ resembled a dislocated fragment.
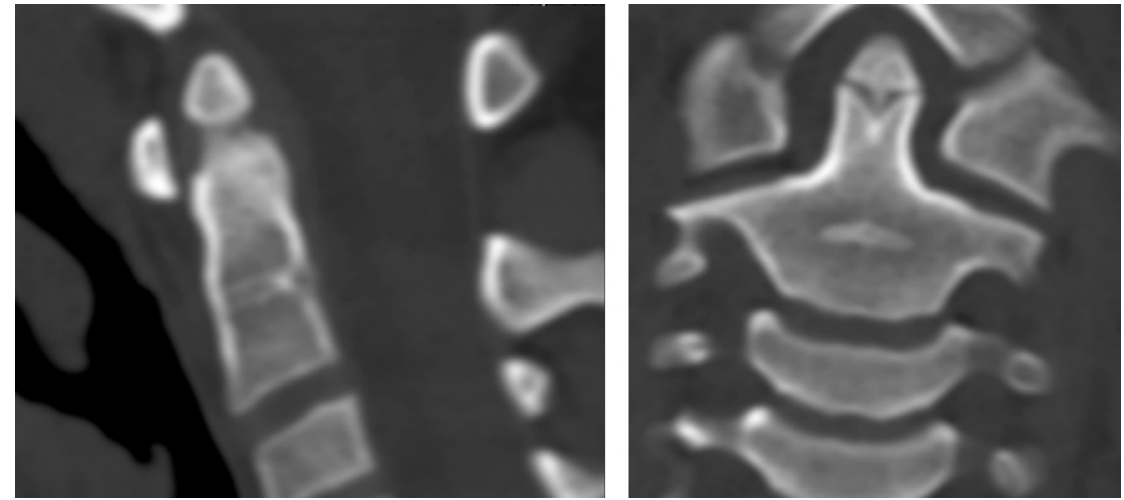

Fig 13. Large ossiculum terminale in an 8-year-old child and incomplete ossification of the ADS.

would have been ideal, but in our cross-sectional approach, we did not actually see the ossification progress or synchondroses close. Furthermore, the degree of maturation was subjective, and neither inter- nor intraobservation analysis was attempted. Finally, as the real progress of ossification could not be directly determined, we arbitrarily defined the "normal" closure age to correspond to the age category in which more than $80 \%$ of the patients showed definitive closure.

\section{Conclusions}

Ossification of the craniovertebral junction is a complex process. In the pediatric population, knowledge of ossification milestones of the atlas and axis are mandatory for discriminating between normal anatomy, variants, and true osseous lesions. Triplanar CT reconstructions should be routinely conducted to enhance diagnostic certainty in the analysis of ossification patterns.

\section{References}

1. Ogden JA. Skeletal Injury in the Child Spine. 2nd ed. Philadelphia: Saunders; 1990:562-571

2. Ogden JA. Radiology of postnatal skeletal development. XI. The first cervical vertebra. Skeletal Radiol 1984;12:12-20

3. Ogden JA, Murphy MJ, Southwick WO, et al. Radiology of postnatal skeletal development. XIII. C1-C2 interrelationships. Skeletal Radiol 1986;15:433-38

4. Müller F, O'Rahilly R. Occipitocervical segmentation in staged human embryos. J Anat 1994;185:251-58

5. Lustrin ES, Karakas SP, Ortiz AO, et al. Pediatric cervical spine: normal anatomy, variants, and trauma. Radiographics 2003;23:539-60

6. Ogden JA. Radiology of postnatal skeletal development. XII. The second cervical vertebra. Skeletal Radiol 1984;12:169-77

7. Calvy TM, Segall HD, Gilles FH, et al. CT anatomy of the craniovertebral junction in infants and children. AJNR Am J Neuroradiol 1987;8:489-94
8. Piatt JH Jr, Grissom LE. Developmental anatomy of the atlas and axis in childhood by computed tomography. J Neurosurg Pediatr 2011;8:235-43

9. Hadley MN, Walters BC, Grabb PA, et al. Guidelines for the management of acute cervical spine and spinal cord injuries. Clin Neurosurg 2002;49:407-98

10. Rajwani T, Bhargava R, Moreau M, et al. MRI characteristics of the neurocentral synchondrosis. Pediatr Radiol 2002;32:811-16

11. Gehweiler JA Jr, Daffner RH, Roberts L Jr. Malformations of the atlas vertebra simulating the Jefferson fracture. AJR Am J Roentgenol 1983;140:1083-86

12. Smoker WR. Craniovertebral junction: normal anatomy, craniometry, and congenital anomalies. Radiographics 1994;14:255-77

13. Haakonsen M, Gudmundsen TE, Histol O. Midline anterior and posterior atlas clefts may simulate a Jefferson fracture. A report of 2 cases. Acta Orthop Scand 1995;66:369-71

14. Scheuer JL, Black SM. The Juvenile Skeleton. London: Academic Press; 2004

15. Geipel P. Studies on the fissure formation of the atlas and epistropheus. IV. [in German] Zentralbl Allg Pathol 1955;94:19-84

16. Swischuk LE. The cervical spine in childhood. Curr Probl Diagn Radiol $1984 ; 13: 1-26$

17. Castellana C, Kosa F. Morphology of the cervical vertebrae in the fetal-neonatal human skeleton. J Anat 1999;194:147-52

18. Wollin DG. The os odontoideum. Separate odontoid process. J Bone Joint Surg Am 1963;45:1459-71

19. Fielding JW, Hensinger RN, Hawkins RJ. Os odontoideum. J Bone Joint Surg Am $1980 ; 62: 376-83$

20. Sankar WN, Wills BP, Dormans JP, et al. Os odontoideum revisited: the case for a multifactorial etiology. Spine (Phila Pa 1976) 2006;31:979-84

21. Amling M, Posl M, Wening VJ, et al. Structural heterogeneity within the axis: the main cause in the etiology of dens fractures. A histomorphometric analysis of 37 normal and osteoporotic autopsy cases. J Neurosurg 1995;83:330-35

22. Roche C, Carty H. Spinal trauma in children. Pediatr Radiol 2001;31:677-700

23. Gebauer M, Lohse C, Barvencik F, et al. Subdental synchondrosis and anatomy of the axis in aging: a histomorphometric study on 30 autopsy cases. Eur Spine J 2006;15:292-98

24. Swischuk LE, John SD, Moorthy C. The os terminale-os odontoideum complex. Emerg Radiol 1997;4:72-81

25. Ogden JA, Ganey TM (2003) Development and maturation of the spine and spinal cord. In: DeWald RL, Arlet V, Carl AL, et al, eds. Spinal Deformities. The Comprehensive Text. New York: Thieme Medical Publishers; 2003: 54-71 